\title{
Investigating the Resistance of Escherichia coli Against Some Selected Antimicrobials in Bam
}

\author{
Jamshid Ayatollahi ${ }^{1}$, Alireza Vahidi ${ }^{2}$, Seyed Hossein Shahcheraghi ${ }^{1, *}$, Alireza Bagheripour ${ }^{3}$, \\ Marzieh Lotfi ${ }^{4}$, Malihe Lotfi ${ }^{5}$, Saeid Reza Lotfi ${ }^{6}$ \\ ${ }_{2}^{1}$ Infectious and Tropical Diseases Research Center, Shahid Sadoughi University of Medical Sciences, Yazd, IR Iran \\ ${ }_{2}^{2}$ Research and Clinical Centre for Infertility, Shahid Sadoughi University of Medical Sciences, Yazd, IR Iran \\ ${ }^{3}$ Medical Department, Shahid Sadoughi University of Medical Sciences, Yazd, IR Iran \\ ${ }^{4}$ Department of Modern Sciences \& Technologies, Faculty of Medicine, Mashhad University of Medical Sciences, Mashhad, IR Iran \\ 5 Department of Biotechnology, Faculty of Medicine, Zanjan University of Medical Sciences, Zanjan, IR Iran \\ ${ }^{6}$ Department of Pediatrics, Samen Alaeme Hospital of Naja, Mashhad, IR Iran \\ *Corresponding author: Seyed Hossein Shahcheraghi, Infectious and Tropical Diseases Research Center, Shahid Sadoughi University of Medical Sciences, Yazd, IR Iran. Tel: +98- \\ 9132531389, E-mail: shahcheraghih@gmail.com.
}

Received: July 24, 2012; Revised: October 29, 2012; Accepted: November 10, 2012

Background: Antibiotics and corresponding resistance genes and resistant bacteria have been considered as emerging pollutants worldwide. Excessive and incorrect use of antimicrobials in human and veterinary medicine, as well as their metaphylactic application in livestock are considered to be key aspects of this current situation.

Objectives: The current study aimed to investigate the resistance of Escherichia coli against six antibiotics, in Bam.

Materials and Methods: This descriptive study was performed on 300 samples with positive cultures of E. coli in 2006-2007 at the Central Laboratory and the Pasteur hospital in Bam city. Information related to this study was first collected by visiting the above laboratories and completing questionnaires and then recorded in the Excel program and analyzed using the SPSS software and chi-square statistical method.

Results: In this study, high resistances were found to nalidixic acid (59.7\%) and gentamicin (52.3\%) but high susceptibilities were found to ciprofloxacin (59.3\%) and ceftriaxone (36.3\%).

Conclusions: According to the results obtained in this study ciprofloxacin is an effective antibiotic in E.coli infections.

Keywords: Escherichia coli; Antibiotics; Bacterial Resistance

\section{Background}

Escherichia coli is a Gram-negative bacillus that belongs to the Escherichia genus, which includes species present in the human and other animal intestine. When eliminated in the environment together with feces it contaminates water, soil and food (1-3). Based on the particular present strain, it can provide resistance against pathogenic organisms or can itself be pathogenic, causing diseases at intestinal and extra-intestinal sites (4). Particularly, urban rivers have been often reported as having fecal contamination associated with antibiotic-resistant E. coli which threatens drinking water quality and public health (5-7).

Nowadays the microbial resistance to antibiotics has caused various problems in the process of disinfecting devices and surfaces of the hospitals and in treatment of the patients (8-10). Extensive clinical use of antibiotics in both human and veterinary medicine has been blamed for the rise and spread of antibiotic-resistant (AR) bac- teria (11). But, in recent years, several reports have raised concerns that antibacterial drug development will not adequately address the problems posed by antibiotic resistance among important bacterial pathogens (12-15). However, the widespread use of antibiotics in animals as well as human beings has resulted in the frequent detection of antibiotic-resistant bacteria in human waste, meat, fish farms, rivers, and lakes $(16,17)$.

To date, the relationship between concentration of antibiotics and development of resistance in bacterial pathogens is often unclear (18). Surveillance data show that resistance in E. coli is consistently the highest to antimicrobial agents that have been in use the longest time in human and veterinary medicine (19).

\section{Objectives}

The present study was designed to isolate E. coli strains from patients to assess the susceptibility and resistance patterns to some selected antimicrobials during 2006 2007.

Implication for health policy/practice/research/medical education:

Our study revealed that the highest resistances were to nalidixic acid and gentamicin whereas the highest susceptibilities were to ciprofloxacin and ceftriaxone. Therefore, ciprofloxacin is an alternative antibiotic in E. coli infections.

Copyright (C 2013, Ahvaz JundishapurUniversity of Medical Sciences; Published by Kowsar Corp. This is an open-access article distributed under the terms of the Creative Commons Attribution License, which permits unrestricted use, distribution, and reproduction in any medium, provided the original work is properly cited. 


\section{Materials and Methods}

This descriptive study was performed on 300 samples with positive cultures of E. coli during 2006-2007 at the Central Laboratory and the Pasteur hospital in Bam city. Standard methods were used for isolation, identification and biochemical confirmation (indole, methyl-red, Voges-Proskauer and Simmons citrate tests) of E. coli isolates (20).

The antibiotic resistance was determined by a standard disc diffusion technique using Mueller-Hinton agar (Merck, Germany) according tothe recommendations of National Commitee for Clinical Laboratory Standards (NCCLS 2008) (21). The antimicrobial drugs were tested and their sensidisk concentrations were: Nalidixic acid (NA, $30 \mu \mathrm{g})$, Ceftizoxime (CT, 30 $\mu \mathrm{g})$, Gentamicin (GM, $10 \mu \mathrm{g})$, Ciprofloxacin (CP, $5 \mu \mathrm{g})$, Ceftriaxone (CEF, $30 \mu \mathrm{g})$, Cotrimoxazole (SUT, $25 \mu \mathrm{g}$ ) (The Antibiotic discs were made by Oxoid Company, South Australia, http://www. oxoid.com/UK/blue/contacts/contacts.asp?compType=0). Within $15 \mathrm{~min}$ of the application of the discs, the plates were inverted and incubated at $37^{\circ} \mathrm{C}$. After $24 \mathrm{~h}$ of incubation, the plates were examined, and the diameters of the zones of complete inhibition to the nearest whole millimeter were measured. The zone diameter for individual antimicrobial agents was then translated into sensitive, intermediate and resistant categories.

The sample types included urine, blood and feces (50 blood samples, 105 urine and 145 fecal samples). Finally, the information was recorded in the Excel program and analyzed using the SPSS software and chi-square statistical method.

\section{Results}

During the study period, 300 culture-positive results were investigated for E. coli. The results related to the sensitivity rate of the bacteria culture-positive samples based on gender in the case of Ciprofloxacin included 178 sensitive cases (59.3\%), 49 (16.3\%) semi-sensitive and 73 resistant cases $(24.3 \%)$. In Ceftriaxone, 109 cases (36.3\%) were sensitive, 105 cases (35\%) semi-sensitive and 86 cases (28.7\%) resistant. In Ceftizoxime, 81 cases (27\%) were sensitive, 134 cases $(44.7 \%)$ semi-sensitive and 85 cases $(28.3 \%)$ resistant. In Co-trimoxazole, 68 cases (22.7\%) were sensitive, 113 cases (37.7\%) semi-sensitive and 119 cases (39.7\%) resistant. In Nalidixic Acid, 46 cases (15.3\%) were sensitive, 75 cases (25\%) semi-sensitive and 179 cases (59.7\%) resistant. In Gentamicin, 34 cases (11.3\%) were sensitive, 109 cases (36.3\%) semi-sensitive and 157 cases (52.3\%) resistant. The results based on gender regarding Ciprofloxacin, Ceftriaxone, Nalidixic acid, Cotrimoxazole and Gentamicin weren't significant with $\mathrm{P}$ value $=0.367$ ، $\mathrm{P}$ value $=0.987$ $\mathrm{P}$ value $=0.353$ ، $\mathrm{P}$ value $=0.347$ and $\mathrm{P}$ value $=0.104$ respec tively but theresult was significant in Ceftizoxime with $\mathrm{P}$ value $=0.042$.

The results related to the sensitivity rate of the bacteria culture-positive samples based on blood, urine and feces samples in the case of Ciprofloxacin included the highest sensitivity to stool culture (62.8\%) and the highest resistance to urine culture (31.4\%). In Ceftriaxone, the highest sensitivity and resistance were to urine culture (37.1\%) and stool culture (31.7\%), respectively. In Ceftizoxime, the highest sensitivity and resistance were related to blood culture (46\%) and stool culture (33.1\%), respectively. In Cotrimoxazole, the highest sensitivity and resistance were related to blood culture (34\%) and stool culture (45.5\%), respectively. In Nalidixic Acid, the highest sensitivity and resistance were related to urine culture (18.1\%) and (63.8\%), respectively. In Gentamicin, the highest sensitivity and resistance were related to stool culture (12.4\%) and (56.2\%), respectively.

The results based on blood, urine and feces samples in Ciprofloxacin, Ceftriaxone, Nalidixic acid and Gentamicin weren't significant with $\mathrm{P}$ value $=0.209$. $\mathrm{P}$ value $=$ 0.56 . $P$ value $=0.205$ and $P$ value $=0.430$, respectively but this result was significant in ceftizoxime and Co-trimoxazole with $\mathrm{P}$ value $=0.07$ and $\mathrm{P}$ value $=0.015$, respectively . Overall in this study, the highest sensitivity and the highest resistance were related to Ciprofloxacin, Ceftriaxone and Nalidixic acid, Gentamicin, respectively (Table 1).

\begin{tabular}{llll}
\hline \multicolumn{1}{l}{ Table 1. Distribution Pattern of $E$. coli Resistance to Various Antibiotics in This Study } \\
\hline Antibiotics & Sensitive, No. (\%) & Semi-sensitive, No. (\%) & Resistant, No. (\%) \\
\hline Ciprofloxacin & $178(59.3)$ & $49(16.3)$ & $73(24.3)$ \\
Ceftriaxone & $109(36.3)$ & $105(35)$ & $86(28.7)$ \\
Ceftizoxime & $81(27)$ & $134(44.7)$ & $85(28.3)$ \\
Co-trimoxazole & $68(22.7)$ & $113(37.7)$ & $119(39.7)$ \\
Nalidixic Acid & $46(15.3)$ & $75(25)$ & $179(59.7)$ \\
Gentamicin & $34(11.3)$ & $109(36.3)$ & $157(52.3)$ \\
\hline
\end{tabular}

\section{Discussion}

Antibiotics and resistant bacteria have been considered as emerging pollutants worldwide. Infectious diseases caused by antibiotic-resistant bacteria have been a serious concern in local hospitals and clinics. The spread of antibiotic-resistant bacteria is an emerging worldwide problem that increasingly threatens the success- 
Ayatollahi J et al.

ful treatment of infectious diseases. Antibiotic-resistant Gram-negative bacteria such as E.coli are the emerging problems that infection control practitioners, hospital epidemiologists, clinicians, and hospital administrators are struggling to control. Therefore, the present study was conducted to determine the antimicrobial resistance of E. coli isolated from patients in Bam.

A study investigated microbial species isolated from patients with urinary tract infection (UTI) and evaluated their in vitro susceptibility patterns to commonly used antimicrobial agents in Karaj, Iran. E. coli isolates, the predominant cause of UTIs, showed high resistance to gentamicin (73.69\%). These isolates, the predominant cause of UTIs, showed high resistance to Gentamicin, Ampicillin and Cephalothin, and higher sensitivity to imipenem, Ciprofloxacin, Nitrofurantoin, and Ceftizoxime (22). The difference between this study and the present study was that in the current study was found to be most susceptible to Ciprofloxacin (59.3\%). Also, in the current study the highest resistances were to Nalidixic acid (59.7\%) and Gentamicin (52.3\%), respectively.

Another study was performed to determine the frequency and antimicrobial susceptibility of $E$. coli pathotypes obtained from children with acute diarrhea. The in vitro antibiotic susceptibility pattern of E. coli strains showed that $36.4 \%$ of isolates were found to be resistant to nalidixic acid. Also, $89.9 \%, 88.9 \%$ and $79.8 \%$ of isolates were resistant to Tetracycline, Chloramphenicol, and Ampicillin, respectively (23). The difference between this study and the present study was that in the current study was found to be most resistance to Nalidixic acid (59.7\%).

A study was conducted to estimate the presence of E. coli in sea foods in the landing centers and local market and their antibiotic resistance was tested with 15 antibiotics for the benefit of the consumers. This study revealed that 91.2\% of E. coli isolates were susceptible to ciprofloxacin (24). This study revealed that amikacin, ciprofloxacin and chloramphenicol are the best antibiotics to treat E. coli infection. Results of the current study also proved high susceptibility to Ciprofloxacin (59.3\%).

A study was conducted to determine the presence or absence of bacterial infections in tracheal tubes and determination of their antimicrobial susceptibility patterns specially E.coli. The results showed that 67.3\% of $E$. coli isolates were resistant to Nalidixic acid (25). The similarity between this study and the present study was that in the current study was found to be most resistance to Nalidixic acid (59.7\%). Therefore, it proved the results of the current study.

Another study was conducted to determine the rate of resistance to antibiotics by E. coli strains isolated from cultures of different clinical specimens received from pediatric patients in Jordan. The high resistance rate was observed toampicillin (84\%), followed by Amoxicillin-Clavulanic acid (74.3\%), Cotrimoxazole (71\%), Nalidixic acid (47.3\%), Cephalothin (41\%). Lower resistance rates were observed Toamikacin (0\%) followed by Cefotaxime (11\%),
Ceftriaxone (11.7\%), Ciprofloxacin (14.5\%), Norfloxacin (16.5\%), Gentamicin (17.3\%), Cephalexin (20.9\%), Ceftazidime (22.5\%), Cefixime (29.6\%), and cefaclor (32.8\%) (26). The current study also proved high susceptibility to ciprofloxacin (59.3\%) and the highest rate of resistance was against nalidixic acid (59.7\%).

In another study, antibiotic susceptibility test was performed for all the isolates in Nepal. Results indicated that out of 3,460 urine samples 680 (19.7\%) showed the significant bacteriuria. The most common isolated pathogens were E. coli $75.7 \%$ followed by Klebsiella pneumoniae 10.7\%, Acinetobacter spp. 5.5\%, Proteus spp. 3.5\% and Pseudomonas aeruginosa $1.2 \%$. The most susceptible antibiotics were Amikacin, Ceftriaxone and Ciprofloxacin for most of the isolates. E. coli which was the main isolate was found to be most susceptible to Amikacin 96.1\%, Nitrofurantoin 91.3\% and Gentamicin 77.7\% followed by Ceftriaxone $65.8 \%$ and Ciprofloxacin 64.1\% (27). The difference between this study and the present study was that in the current study was found to be most susceptible to Ciprofloxacin $59.3 \%$.

According to the above studies, the investigation of bacterial resistance against antibiotics is essential and the present study was performed on E. coli. Results of the current study revealed that the highest resistances were to nalidixic acid and gentamicin. In addition, it was proved that the highest susceptibilities were to Ciprofloxacin and Ceftriaxone. Therefore, Ciprofloxacin is an alternative antibiotic in E.coli infections.

\section{Acknowledgements}

We thank the personnel of Central Laboratory and the Pasteur hospital in Bam for their cooperation in completing the necessary data for this study.

\section{Authors Contribution}

None declared.

\section{Financial Disclosure}

None declared.

\section{Funding/Support}

None declared.

\section{References}

1. Sodha SV, Lynch M, Wannemuehler K, Leeper M, Malavet M, Schaffzin J, et al. Multistate outbreak of Escherichia coli O157:H7 infections associated with a national fast-food chain, 2006: a study incorporating epidemiological and food source traceback results. Epidemiol Infect. 2011;139(2):309-16.

2. Taur Y, Smith MA. Adherence to the Infectious Diseases Society of America guidelines in the treatment of uncomplicated urinary tract infection. Clin Infect Dis. 2007;44(6):769-74.

3. von Baum H, Marre R. Antimicrobial resistance of Escherichia coli and therapeutic implications. Int JMed Microbiol. 2005;295(67):503-11.

4. Bailey MT, Engler H, Sheridan JF. Stress induces the translocation of cutaneous and gastrointestinal microflora to secondary 
lymphoid organs of C57BL/6 mice. J Neuroimmunol. 2006;171(12):29-37.

5. Hu J, Shi J, Chang H, Li D, Yang M, Kamagata Y. Phenotyping and genotyping of antibiotic-resistant Escherichia coli isolated from a natural river basin. Environ Sci Technol. 2008;42(9):3415-20.

6. Servais P, Passerat J. Antimicrobial resistance of fecal bacteria in waters of the Seine river watershed (France). Sci Total Environ. 2009;408(2):365-72.

7. Watkinson AJ, Micalizzi GB, Graham GM, Bates JB, Costanzo SD. Antibiotic-resistant Escherichia coli in wastewaters, surface waters, and oysters from an urban riverine system. Appl Environ Microbiol. 2007;73(17):5667-70.

8. Kasra Kermanshahi R, Kazemi M, Heshmat Dehkordi E, Payami F. The study of influence of some physicochemical agents in resistance pattern of Escherichia coli and Pseudomonas aeruginosa. Jundishapur J Microbiol. 2011;4(4):239-247.

9. Klevens RM, Morrison MA, Nadle J, Petit S, Gershman K, Ray S, et al. Invasive methicillin-resistant Staphylococcus aureus infections in the United States. JAMA. 2007;298(15):1763-71.

10. Rosenblatt-Farrell N. The landscape of antibiotic resistance. Environ Health Perspect. 2009;117(6):A244-50.

11. Levy SB. Antibiotic resistance: consequences of inaction. Clin Infect Dis. 2001;33 Suppl 3:S124-9.

12. Cars O, Hogberg LD, Murray M, Nordberg O, Sivaraman S, Lundborg CS, et al. Meeting the challenge of antibiotic resistance. BMJ. 2008;337:a1438.

13. Nathan C. Antibiotics at the crossroads. Nature 2004;431(7011):899-902.

14. Norrby SR, Nord CE, Finch R. Lack of development of new antimicrobial drugs: a potential serious threat to public health. Lancet Infect Dis. 2005;5(2):115-9.

15. Spellberg B, Powers JH, Brass EP, Miller LG, Edwards JE, Jr. Trends in antimicrobial drug development: implications for the future. Clin Infect Dis. 2004;38(9):1279-86.

16. Ash RJ, Mauck B, Morgan M. Antibiotic resistance of Gramnegative bacteria in rivers, United States. Emerg Infect Dis. 2002;8(7):713-6.
17. Iwane T, Urase T, Yamamoto K. Possible impact of treated wastewater discharge on incidence of antibiotic resistant bacteria in river water. Water Sci Technol. 2001;43(2):91-9.

18. Subbiah M, Mitchell SM, Ullman JL, Call DR. beta-lactams and florfenicol antibiotics remain bioactive in soils while ciprofloxacin, neomycin, and tetracycline are neutralized. Appl Environ Microbiol. 2011;77(20):7255-60.

19. Tadesse DA, Zhao S, Tong E, Ayers S, Singh A, Bartholomew MJ, et al. Antimicrobial drug resistance in Escherichia coli from humans and food animals, United States, 1950-2002. Emerg Infect Dis. 2012;18(5):741-9.

20. Dragan-Bularda M. [General Microbiology - a laboratory manual]. 3 ed: Romania; 2000

21. Performance standards for antimicrobial disk and dilution susceptibility tests for bacteria isolated from animals. approved standards, M2-A9, Tabel 2A. 2008;27(1):32-37.

22. Khoshbakht R, Salimi A, Shirzad Aski H, Keshavarzi H. Antibiotic Susceptibility of Bacterial Strains Isolated From Urinary Tract Infections in Karaj, Iran. JundishapurJ Microbiol. 2013;6(1):86-90.

23. Kalantar E, Soheili F, Salimi H, Soltan Dallal MM. Frequency, antimicrobial susceptibility and plasmid profiles of Escherichia coli pathotypes obtained from children with acute diarrhea. JundishapurJMicrobiol. 2012;4(1):23-28.

24. Jeyasanta KI, Aiyamperumal V, Patterson J. Prevalence of antibiotic resistant Escherichia coli in sea foods of Tuticorin coast, Southeastern India. Adv Biological Res. 2012;6:70-77.

25. Khosravi A, Parhizgari N, Montazeri E, Mozaffari A, Abbasi F. The Prevalence of Bacteria Isolated From Endotracheal Tubes of Patients in Golestan Hospital, Ahvaz, Iran, and Determination of Their Antibiotic Susceptibility Patterns. Jundishapur J Microbiol. 2013;6(1):67-71.

26. Alshara M. Antimicrobial resistant pattern of Escherichia coli strains isolated from pediatric patients in Jordan. Acta Med Iran. 2011;49(5):293-5.

27. Raza S, Pandey S, Bhatt CP. Microbiological analysis of isolates in Kathmandu Medical College Teaching Hospital, Kathmandu, Nepal. Kathmandu Univ Med J(KUMJ). 2011;9(36):295-7. 